Research Article

\title{
Sodium Butyrate-Modulated Mitochondrial Function in High-Insulin Induced HepG2 Cell Dysfunction
}

\author{
Tingting Zhao, ${ }^{1}$ Junling Gu, ${ }^{1}$ Huixia Zhang, ${ }^{1}$ Zhe Wang, ${ }^{1}$ Wenqian Zhang, ${ }^{1}$ Yonghua Zhao, ${ }^{2}$ \\ Ying Zheng, ${ }^{2}$ Wei Zhang $\mathbb{D}^{1},{ }^{1}$ Hua Zhou $\mathbb{D}^{1,},{ }^{1,3}$ Guilin Zhang, ${ }^{1}$ Qingmin Sun, ${ }^{4}$ Enchao Zhou ${ }^{\mathbb{D}},{ }^{4}$ \\ Zhilong Liu $\mathbb{D}^{3}$ and Youhua $\mathrm{Xu} \mathbb{D}^{1,3}$ \\ ${ }^{1}$ Faculty of Chinese Medicine, State Key Laboratory of Quality Research in Chinese Medicine, Macau University of Science \\ and Technology, Taipa, Macao, China \\ ${ }^{2}$ Institute of Chinese Medical Sciences, State Key Laboratory of Quality Research in Chinese Medicine, University of Macau, Taipa, \\ Macao, China \\ ${ }^{3}$ Department of Endocrinology, Zhuhai Hospital of Integrated Traditional Chinese and Western Medicine, Zhuhai, China \\ ${ }^{4}$ Jiangsu Province Hospital of Traditional Chinese Medicine, Affiliated Hospital of Nanjing University of Chinese Medicine, \\ Hanzhong Road, Nanjing, China
}

Correspondence should be addressed to Enchao Zhou; zhouenchao@njucm.edu.cn, Zhilong Liu; zhuhaizhilong@163.com, and Youhua Xu; yhxu@must.edu.mo

Received 27 April 2020; Accepted 1 June 2020; Published 17 July 2020

Guest Editor: Yue Liu

Copyright ( 2020 Tingting Zhao et al. This is an open access article distributed under the Creative Commons Attribution License, which permits unrestricted use, distribution, and reproduction in any medium, provided the original work is properly cited.

\begin{abstract}
The liver plays a pivotal role in maintaining euglycemia. Biogenesis and function of mitochondria within hepatocytes are often the first to be damaged in a diabetic population, and restoring its function is recently believed to be a promising strategy on inhibiting the progression of diabetes. Previously, we demonstrated that the gut microbiota metabolite butyrate could reduce hyperglycemia and modulate the metabolism of glycogen in both $\mathrm{db} / \mathrm{db}$ mice and HepG2 cells. To further explore the mechanism of butyrate in controlling energy metabolism, we investigated its influence and underlying mechanism on the biogenesis and function of mitochondria within high insulin-induced hepatocytes in this study. We found that butyrate significantly modulated the expression of 54 genes participating in mitochondrial energy metabolism by a PCR array kit, both the content of mitochondrial DNA and production of ATP were enhanced, expressions of histone deacetylases 3 and 4 were inhibited, betaoxidation of fatty acids was increased, and oxidative stress damage was ameliorated at the same time. A mechanism study showed that expression of GPR43 and its downstream protein beta-arrestin2 was increased on butyrate administration and that activation of Akt was inhibited, while the AMPK-PGC-1alpha signaling pathway and expression of p-GSK3 were enhanced. In conclusion, we found in the present study that butyrate could significantly promote biogenesis and function of mitochondria under high insulin circumstances, and the GPR43- $\beta$-arrestin2-AMPK-PGC1-alpha signaling pathway contributed to these effects. Our present findings will bring new insight on the pivotal role of metabolites from microbiota on maintaining euglycemia in diabetic population.
\end{abstract}

\section{Introduction}

Type 2 diabetes (T2D) has become a major threat to health worldwide. It is estimated that the diabetic population will rise to 600 million people within the next 20 years, accounting for about $10 \%$ of the world population. The liver plays a pivotal role in maintaining euglycemia; unfortunately, as high as $19 \%$ of cases with type 2 diabetes are reported being accompanied with liver dysfunction [1].

The liver is one of the main target organs for insulin. By modulating glycogenesis or glucose oxidation within hepatocytes, blood glucose is maintained in a relatively stable state. However, a very high level of insulin, or the so-called insulin resistance (IR), will significantly destroy the capacity of the 
TABLE 1: List of sequences for primers used in PCR analysis.

\begin{tabular}{lrr}
\hline & Forward primers $\left(5^{\prime}\right.$ to $\left.3^{\prime}\right)$ & Reverse primers $\left(5^{\prime}\right.$ to $\left.3^{\prime}\right)$ \\
\hline GLUT2 & GACAGTGAAAACCAGGGTCC & TGTGCCACACTCACACAAGA \\
GLUT4 & GCCCTAACTTTCTTCCTCTCCCT & CCGACCTTTGGTTTCTTCTCTCA \\
HDAC3 & CTGTGTAACGCGAGCAGAAC & GCAAGGCTTCACCAAGAGTC \\
HDAC4 & CTGGTCTCGGCCAGAAAGT & CGTGGAAATTTGAGCCATT \\
ACADS & CCCATCTTCTTCACCTGAGC & ACACACCAGATGTTGCTCCA \\
HADH & ACCCTGAGCACCATAGCGA & CAGCGAATCGGTCTTGTCTGG \\
CPT1A & ATCAATCGGACTCTGGAAACGG & TCAGGGAGTAGCGCATGGT \\
n-Actin & GTTGTCGACGACGAGCG & GCACAGAGCCTCGCCTT \\
mDNA & TGAGGCCAAATATCATTCTGAGGGGC & TTTCATCATGCGGAGATGTTGGATGG \\
MinArc & ACATGATTAGCAAAAGGGCCTAGCTTGGACTCAGA & TGCACCTGCTCTGTGATTATGACTATCCCACAGTC \\
\hline
\end{tabular}

liver in this aspect, and the function and biogenesis of mitochondria are often the first to be damaged $[2,3]$. In this sense, restoring the function of mitochondria is pivotal to inhibit the progression of T2D.

With the understanding of the important role of gut microbiota in disease development, interests have focused on exploring the mechanism of a potential target for controlling T2D. In 2012, Qin and colleagues firstly demonstrated that butyrate-producing bacteria were significantly reduced in a T2D population [4]. Thereafter, studies suggested the potential role of butyrate supplementation on modulating diabetes $[5,6]$. Previously, we demonstrated in $\mathrm{db} / \mathrm{db}$ mice that oral administration with sodium butyrate $(\mathrm{NaB})$ significantly reduced $\mathrm{HbAlc}$ and diabetic inflammation [7]; more importantly, hypertrophy and steatosis of hepatocytes in $\mathrm{db} / \mathrm{db}$ mice were significantly reversed by $\mathrm{NaB}$, accompanied with enhancement of glycogen metabolism [8]. To further investigate the potential role of $\mathrm{NaB}$ on mitochondria, we carried out a series of experiments to observe both the biogenesis and function of mitochondria under high insulin circumstances in this study; the underlying mechanism was also explored. Our present study may bring new insight on understanding the pivotal role of metabolites from microbiota in controlling energy metabolism.

\section{Materials and Methods}

2.1. Materials. Sodium butyrate $(\mathrm{NaB})$ was provided by Meilun Biological Technology (Dalian, China). Antibodies or agents for GAPDH (sc-47724), GPR43 (sc-32906), $\beta$ arrestin2 (sc-13140), Akt (sc-514032), p-Akt (sc-8312), GSK $3 \alpha / \beta$ (sc-7291), p-GSK3 $\alpha / \beta$ (sc-81496), GPR43-siRNA (sc-77339), control siRNA-A (sc-37007), DCFH (sc359840), and JC-1 iodide(sc-364116) were purchased from Santa Cruz (Dallas, TX); AMPK (5832s) and p-AMPK (2531s) were purchased from Cell Signaling Technology (Danvers, MA); PGC1-alpha (ab54481) was purchased from Abcam (Cambridge, UK); and insulin receptor (bs-0681R) was purchased from BIOSS (Greater Boston, New England).
The QIAamp ${ }^{\circledR}$ DNA Micro Kit (56304) and RT ${ }^{2}$ Profiler $^{\mathrm{TM}}$ PCR Array Human Mitochondrial Energy Metabolism (330231) were from QIAGEN (Hilden, Germany); LongAmp $^{\circledR}$ Taq 2X Master Mix (M0287S) was from New England Biolabs (Hitchin, Hertfordshire); and DNA Gel Loading Dye (6X) (R0611), SYBR ${ }^{\mathrm{TM}}$ Safe DNA Gel Stain (S33102), and MitoTracker ${ }^{\mathrm{TM}}$ Deep Red FM (M22426) were from Thermo Scientific (Massachusetts, US). 1-Step Quantitative Reverse Transcription PCR (RT-qPCR) from RNA (1725151) was from BIO-RAD (California, US). ReverTra Ace ${ }^{\circledR}$ qPCR RT Master Mix (FSQ-201) was from Toyobo (Osaka, Japan). Detection kits for ATP, GPX, SOD, and MDA were supplied by Beyotime (Shanghai, China). Kits for NOX2 (SED308Hu) and ACACa (SEB284Hu) were derived from Youersheng (Wuhan, Hubei, China). All other reagents were from commercial sources.

2.2. Cell. HepG2 cells (hepatocyte cell line) were purchased from the American Type Culture Collection (ATCC, Manassas, VA). The cells were cultured in high-glucose MEM medium (Gibco) supplemented with $10 \%$ fetal bovine serum (FBS) and $1 \%$ penicillin-streptomycin at $37^{\circ} \mathrm{C}$ in a $95 \%$ air/5\% $\mathrm{CO}_{2}$ cell incubator.

2.3. Integration of Protein-Protein Interaction Network Analysis. The STRING database (https://string-db.org/) is applied to predict possible interactions among proteins according to the function and pathway enrichment analysis.

2.4. DNA Fragmentation Observation. HepG2 cells were seeded in 6-well plates and treated with insulin or NaB. Total DNA was purified using the DNA extraction kit, separated by $1 \%$ agarose gel, and finally visualized using a GelDoc ${ }^{\mathrm{TM}} \mathrm{XR}+$ imaging system (Bio-Rad, Philadelphia, PA, USA).

2.5. Quantitative Real-Time PCR (Q-PCR). Total RNA from HepG2 cells treated with insulin or $\mathrm{NaB}$ for $24 \mathrm{~h}$ were extracted using a TRIzol reagent according to the manufacturer's protocol. Concentration of RNA was determined by a NanoDrop 2000 instrument (Bio-Rad, USA). cDNA was reverse-transcripted from RNA by a cDNA synthesis kit 
TABLE 2: Expression of mitochondrial energy metabolism-related genes between insulin resistance (insulin) and insulin resistance+sodium butyrate $(\mathrm{NaB} /$ insulin) treatment groups.

\begin{tabular}{|c|c|c|c|c|}
\hline Gene & Accession no. & Normalized ratio (insulin $+\mathrm{NaB} /$ insulin) & $p$ value & Up/downregulation \\
\hline ATP12A & NM_001185085.1 & 1.2014 & 0.0531 & - \\
\hline ATP4A & NM_000704.3 & 0.1417 & 0.0006 & Down \\
\hline ATP4B & NM_000705.4 & 0.0654 & 0.0004 & Down \\
\hline ATP5A1 & NM_001001935.3 & 0.4648 & 0.1675 & Down \\
\hline ATP5B & NM_001686.4 & 0.2171 & 0.0001 & Down \\
\hline ATP5C1 & NM_001001973.3 & 0.0827 & 0.0004 & Down \\
\hline ATP5F1 & NM_001688.5 & 0.6247 & 0.0002 & - \\
\hline ATP5G1 & NM_005175.3 & 1.1209 & 0.4700 & - \\
\hline ATP5G2 & NM_001330269.1 & 0.1045 & $<0.0001$ & Down \\
\hline ATP5G3 & NM_001190329.2 & 0.0006 & $<0.0001$ & Down \\
\hline ATP5H & NM_006356.3 & 0.3197 & 0.0593 & Down \\
\hline ATP5I & NM_007100.4 & 0.0655 & $<0.0001$ & Down \\
\hline ATP5J & NM_001003703.1 & 3.1998 & 0.0354 & Up \\
\hline ATP5J2 & NM_004889.5 & 9.8811 & 0.0006 & Up \\
\hline ATP5L & NM_006476.5 & 0.5051 & $<0.0001$ & - \\
\hline ATP5O & NM_001697.3 & 0.6247 & 0.0002 & - \\
\hline ATP6V0A2 & NM_012463.4 & 0.6408 & 0.0006 & - \\
\hline ATP6V0D2 & NM_152565.1 & 0.6247 & 0.0002 & - \\
\hline ATP6V1C2 & NM_001039362.2 & 2.5339 & 0.0187 & Up \\
\hline ATP6V1E2 & NM_001318063.2 & 0.5028 & 0.0212 & Down \\
\hline ATP6V1G3 & NM_001320218.1 & 1.2351 & 0.5168 & - \\
\hline BCS1L & NM_001079866.2 & 0.7551 & 0.8782 & - \\
\hline COX4I1 & NM_001861.6 & 0.6543 & 0.1425 & - \\
\hline COX4I2 & NM_032609.3 & 13.1592 & $<0.0001$ & Up \\
\hline COX5A & NM_004255.4 & 0.7621 & 0.0348 & - \\
\hline COX5B & NM_001862.3 & 1.3146 & 0.3899 & - \\
\hline COX6A1 & NM_004373.4 & 0.0004 & $<0.0001$ & Down \\
\hline COX6A2 & NM_005205.4 & 2.7473 & 0.0264 & Up \\
\hline COX6B1 & NM_001863.5 & 0.0837 & $<0.0001$ & Down \\
\hline COX6B2 & NM_001369798.1 & 0.0006 & $<0.0001$ & Down \\
\hline COX6C & NM_004374.4 & 0.6320 & 0.0003 & Down \\
\hline COX7A2 & NM_001865.4 & 22.0800 & 0.0001 & Up \\
\hline COX7A2L & NM_004718.4 & 0.0008 & $<0.0001$ & Down \\
\hline COX7B & NM_001866.3 & 0.7817 & 0.0019 & - \\
\hline COX8A & NM_004074.3 & 5.4945 & 0.0001 & Up \\
\hline $\mathrm{COX} 8 \mathrm{C}$ & NM_182971.3 & 3.6587 & $<0.0001$ & Up \\
\hline $\mathrm{CYC1}$ & NM_001916.5 & 0.0004 & $<0.0001$ & Down \\
\hline LHPP & NM_022126.4 & 0.6247 & 0.0002 & - \\
\hline NDUFA1 & NM_004541.4 & 1.3579 & 0.0354 & - \\
\hline NDUFA10 & NM_001322019.1 & 0.6247 & 0.0002 & - \\
\hline NDUFA11 & NM_001193375.2 & 0.7745 & 0.0059 & - \\
\hline NDUFA2 & NM_002488.5 & 2.2212 & 0.0970 & - \\
\hline NDUFA3 & NM_004542.4 & 1.2757 & 0.0033 & - \\
\hline NDUFA4 & NM_002489.4 & 2.8706 & $<0.0001$ & - \\
\hline NDUFA5 & NM_001291304.1 & 0.6247 & 0.0002 & - \\
\hline NDUFA6 & NM_002490.6 & 2.8050 & 0.0002 & Up \\
\hline NDUFA7 & NM_005001.5 & 0.6758 & 0.0504 & - \\
\hline NDUFA8 & NM_001318195.2 & 7.9521 & 0.0023 & Up \\
\hline
\end{tabular}


TABLE 2: Continued.

\begin{tabular}{|c|c|c|c|c|}
\hline Gene & Accession no. & Normalized ratio (insulin $+\mathrm{NaB} /$ insulin) & $p$ value & Up/downregulation \\
\hline NDUFAB1 & NM_005003.3 & 19.8537 & 0.0002 & $\mathrm{Up}$ \\
\hline NDUFB10 & NM_004548.3 & 73.5847 & 0.0001 & Up \\
\hline NDUFB2 & NM_004546.3 & 0.0001 & 0.0001 & Down \\
\hline NDUFB3 & NM_001257102.2 & 0.0657 & 0.0044 & Down \\
\hline NDUFB4 & NM_001168331.2 & 0.0005 & 0.0001 & Down \\
\hline NDUFB5 & NM_002492.4 & 0.0008 & $<0.0001$ & Down \\
\hline NDUFB6 & NM_002493.5 & 0.8693 & 0.0576 & - \\
\hline NDUFB7 & NM_004146.6 & 0.0003 & $<0.0001$ & Down \\
\hline NDUFB8 & NM_005004.4 & 0.5351 & 0.1035 & - \\
\hline NDUFB9 & NM_005005.3 & 0.0035 & 0.0001 & Down \\
\hline NDUFC1 & NM_001184986.1 & 0.0473 & 0.0001 & Down \\
\hline NDUFC2 & NM_004549.6 & 0.3450 & $<0.0001$ & Down \\
\hline NDUFS1 & NM_005006.7 & 0.6176 & 0.0039 & - \\
\hline NDUFS2 & NM_004550.4 & 0.0031 & $<0.0001$ & Down \\
\hline NDUFS3 & NM_004551.3 & 0.6247 & 0.0002 & - \\
\hline NDUFS4 & NM_002495.4 & 0.3749 & $<0.0001$ & Down \\
\hline NDUFS5 & NM_004552.3 & 0.6247 & 0.0002 & - \\
\hline NDUFS6 & NM_004553.6 & 0.1052 & $<0.0001$ & Down \\
\hline NDUFS7 & NM_024407.5 & 2.1856 & 0.0002 & $\mathrm{Up}$ \\
\hline NDUFS8 & NM_002496.4 & 0.6247 & 0.0002 & - \\
\hline NDUFV1 & NM_007103.4 & 0.0424 & $<0.0001$ & Down \\
\hline NDUFV2 & NM_021074.5 & 23.3389 & $<0.0001$ & $\mathrm{Up}$ \\
\hline NDUFV3 & NM_021075.4 & 0.1932 & $<0.0001$ & Down \\
\hline OXA1L & NM_005015.5 & 0.0301 & 0.0001 & Down \\
\hline PPA1 & NM_021129.4 & 0.5502 & 0.0019 & - \\
\hline PPA2 & NM_176869.3 & 0.0111 & $<0.0001$ & Down \\
\hline SDHA & NM_004168.4 & 0.1832 & 0.0008 & Down \\
\hline SDHB & NM_003000.3 & 26.9336 & $<0.0001$ & Up \\
\hline SDHC & NM_003001.5 & 0.0785 & 0.0005 & Down \\
\hline SDHD & NM_003002.4 & 2.8905 & $<0.0001$ & Up \\
\hline UQCR11 & NM_006830.4 & 5.6752 & 0.0049 & Up \\
\hline UQCRC1 & NM_003365.3 & 90.3843 & $<0.0001$ & Up \\
\hline UQCRC2 & NM_003366.4 & 0.1332 & 0.0119 & Down \\
\hline UQCRFS1 & NM_006003.3 & 4.9178 & $<0.0001$ & Up \\
\hline UQCRH & NM_006004.4 & 1.8506 & $<0.0001$ & - \\
\hline UQCRQ & NM_014402.5 & 0.0390 & $<0.0001$ & Down \\
\hline
\end{tabular}

according to the protocol from the supplier as follows: priming for $5 \mathrm{~min}$ at $25^{\circ} \mathrm{C}$, reverse transcription for $20 \mathrm{~min}$ at $46^{\circ} \mathrm{C}$, and RT inactivation for $1 \mathrm{~min}$ at $95^{\circ} \mathrm{C}$. Real-time PCR was performed by FastStart Universal SYBR Green Master. Each sample was mixed with $10 \mu \mathrm{l}$ SYBR master mix, $2 \mu \mathrm{l}$ primers (mixture with both forward and reverse primers), $0.1 \mu \mathrm{l} \mathrm{cDNA}$, and DEPC-treated water to make up a total reaction volume of $20 \mu \mathrm{l}$. Mixtures were circulated for 40 cycles using a high-productivity real-time quantitative PCR ViiATM7 (Life Technologies, Gaithersburg, MD, USA). The reference gene was $\beta$-actin. Each experiment was repeated for at least three times. Sequences for primers used in PCR analysis are listed in Table 1.
2.6. PCR Array Analysis. Quantitative PCR array analysis was carried out using an $\mathrm{RT}^{2}$ Profiler ${ }^{\mathrm{TM}}$ PCR Array Human Mitochondrial Energy Metabolism (QIAGEN). HepG2 cells were treated with high insulin or high insulin $+\mathrm{NaB}$ as indicated. Total RNA was extracted by TRIzol; cDNA was prepared from purified RNA using a ReverTra Ace ${ }^{\circledR}$ qPCR RT Master Mix (FSQ-201, Toyobo); the PCR array assay was analyzed by the kit using the high-productivity real-time quantitative PCR ViiATM7 (Life Technologies, Gaithersburg, MD, USA) according to the manufacturer's instruction. After data collection, relative gene expression was presented as $\Delta \mathrm{Ct}=$ $\mathrm{Ct}(\mathrm{GOI})$ - ave Ct (HKG); the fold change in the gene expression was calculated using the $2^{-\Delta \Delta \mathrm{Ct}}$ method. 


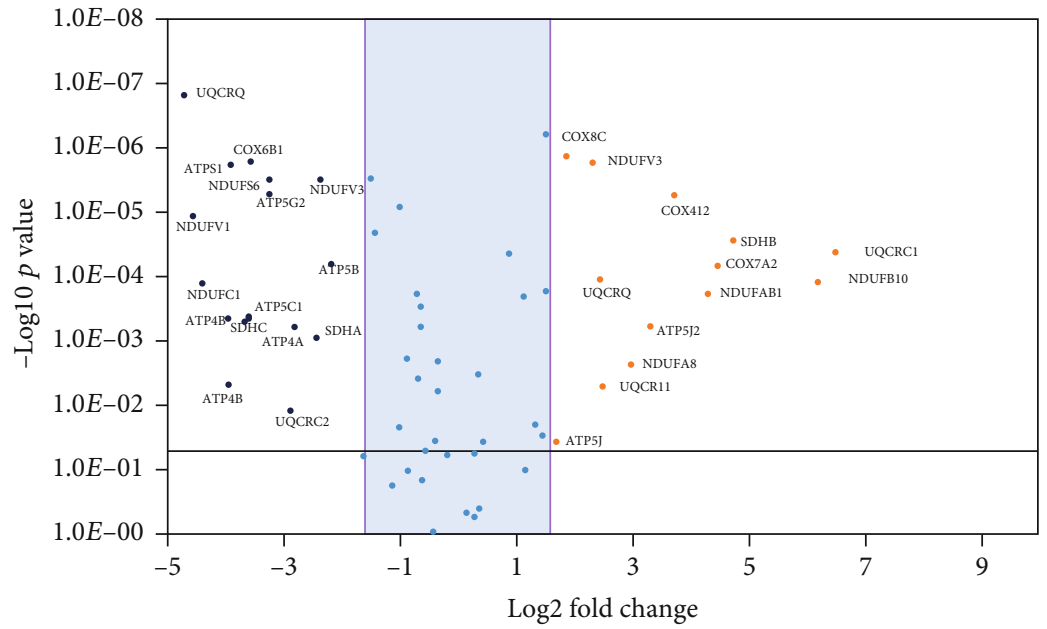

(a)

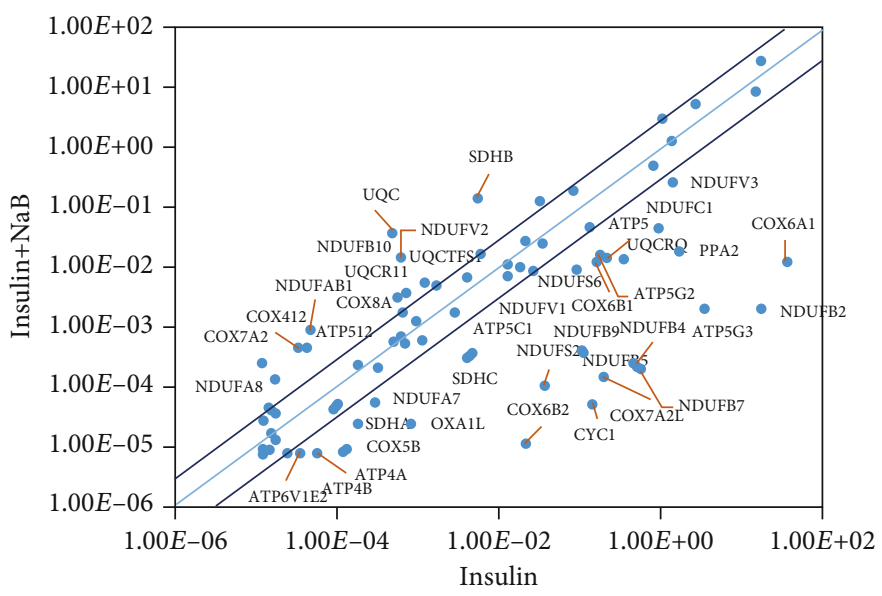

(b)

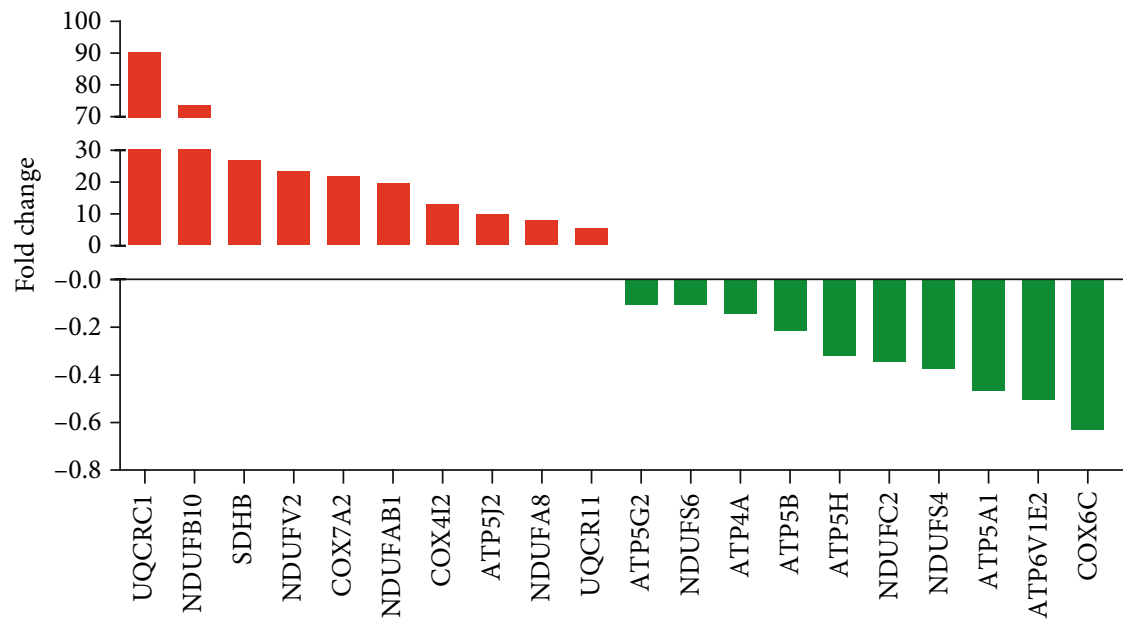

(c)

FIgURe 1: Continued. 


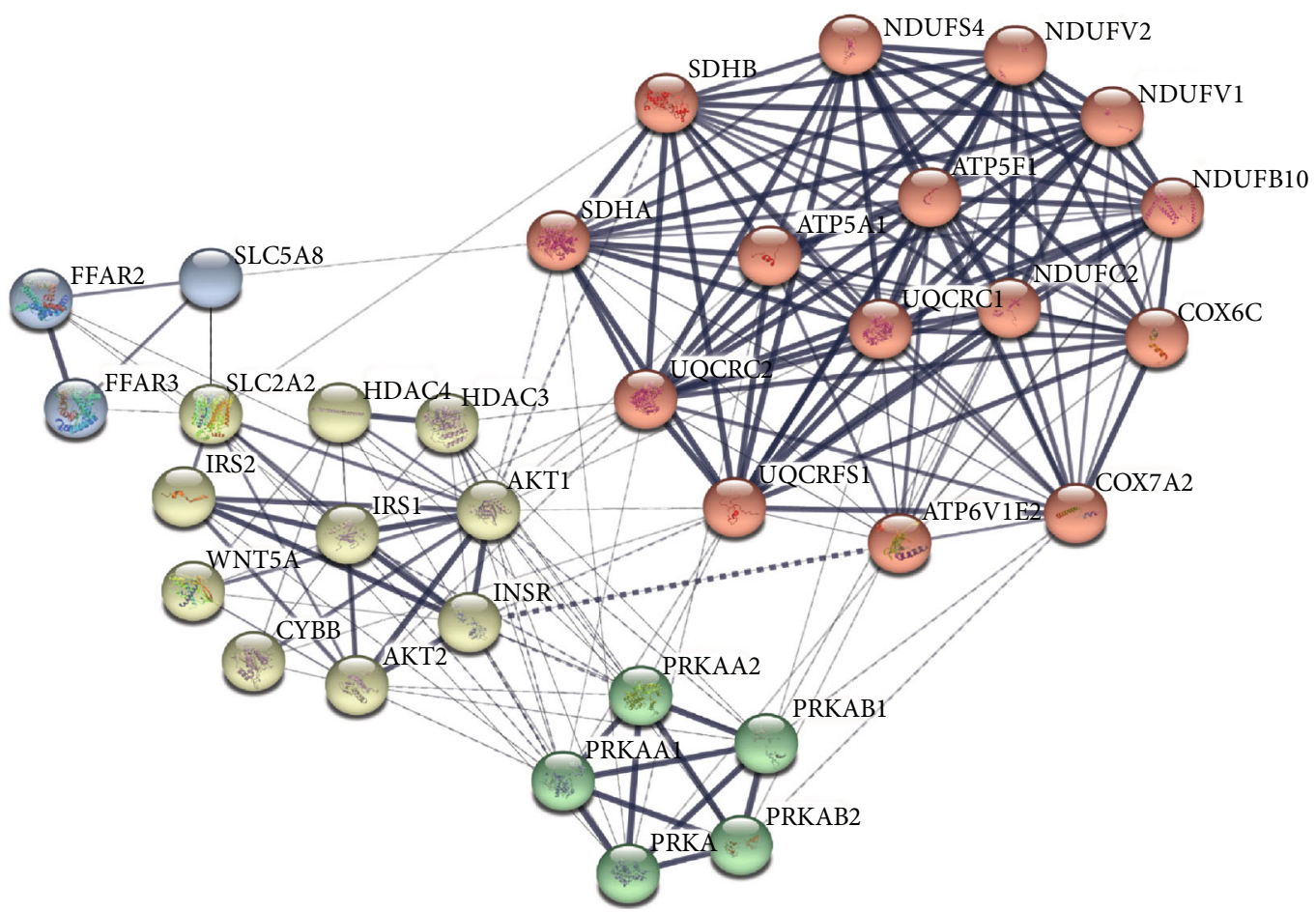

(d)

FIgURE 1: Changes in expression for mitochondrial energy metabolism-related genes between insulin resistance and insulin resistance + sodium butyrate $(\mathrm{NaB})$ treatment groups. $(\mathrm{a}-\mathrm{c})$ Changes for genes related to mitochondrial energy metabolism were assayed by the $\mathrm{RT}^{2}$ Profiler PCR Array; (a) volcano plot for log2 fold changes in genes between groups; (b) relative expression comparison for genes between high insulin ( $x$-axis) and high insulin $+\mathrm{NaB}$ treatment $(y$-axis) groups; (c) fold changes in gene expression for twenty representative genes after $\mathrm{NaB}$ administration. (d) Predicted protein-protein interaction among genes from mitochondrial energy metabolism and genes with $\mathrm{NaB}$ activity generated from the STRING database.

2.7. Flow Cytometry. HepG2 cells $\left(1.5 \times 10^{5}\right.$ cells/well $)$ were seeded in a 6-well plate and administrated with insulin $(0.1 \mu \mathrm{M})$ or $\mathrm{NaB}(0.5 \mathrm{mM})$ for $24 \mathrm{~h}$. Cells were harvested and suspended with PBS solution. Then, the cells were stained with deep red mitochondria $(50 \mathrm{nM}), \mathrm{DCFH}$ $(10 \mu \mathrm{M})$, JC-1 iodide $(2.5 \mu \mathrm{g} / \mathrm{ml})$, or 2 -NBDG $(100 \mu \mathrm{M})$ for $15 \mathrm{~min}$ at room temperature in the dark. The subpopulation of cells was estimated with a BD Aria III Flow Cytometer (BD Biosciences, San Jose, California, USA).

2.8. Knockdown of GPR43. Expressions of GPR43 in HepG2 cells were knocked down according to the protocol from the provider. In general, Lipofectamine ${ }^{\circledR}$ RNAiMAX (13778150) and GPR43 siRNA (sc-77339) were diluted in an Opti-MEM ${ }^{\circledR}$ Medium as instructed from the protocol and then were mixed at the ratio of $1: 1$. The siRNA-lipid mixture was incubated for 10 minutes at room temperature and then cocultured with the cells for 1-3 days within the cell incubator at $37^{\circ} \mathrm{C}$.

2.9. Mitochondrial Imaging. HepG2 cells were incubated with the MitoTracker ${ }^{\mathrm{TM}}$ Deep Red staining solution $(50 \mathrm{nM})$ in the dark for 20 minutes. After being washed with PBS, the mitochondria were observed under a laser confocal microscope (Leica TCS SP8, Germany).
2.10. Immunofluorescence Assay. Cells at the exponential state were incubated with insulin or $\mathrm{NaB}$. Twenty-four hours later, cells were treated with $4 \%$ paraformaldehyde for $30 \mathrm{~min}$. The cells were then blocked with 5\% BSA and incubated with primary antibodies including GPR432 (1:200), insulin receptor $(1: 200)$, p-AKT $(1: 200)$, AKT $(1: 200)$, p-GSK3 $(1: 200)$, GSK3 $(1: 200)$, PGC1- $\alpha(1: 200)$, AMPK $(1: 200)$, p-AMPK $(1: 200)$, or $\beta$-arrestin2 $(1: 200)$ at $4^{\circ} \mathrm{C}$ overnight. After being gently washed with PBS, cells were further incubated with FITC- or CY3-conjugated secondary antibody. The nucleus was stained with DAPI. Finally, the cells were observed under a confocal laser scanning microscope (Leica TCS SP8, Germany), and the fluorescent density was determined by ImageJ software.

2.11. Enzyme Immunoassay (EIA). Levels of malondialdehyde (MDA), glutathione peroxidase (GPX), superoxide dismutase (SOD), NOX2, adenosine triphosphate (ATP), and ACACa were determined by kits according to the manufacturers' protocols.

2.12. Statistical Analysis. All data were obtained from more than three independent repeated experiments and were analyzed by GraphPad Prism 5 software; data that fit into the normal distribution were expressed as mean \pm standard deviation (SD), and the differences among groups were 


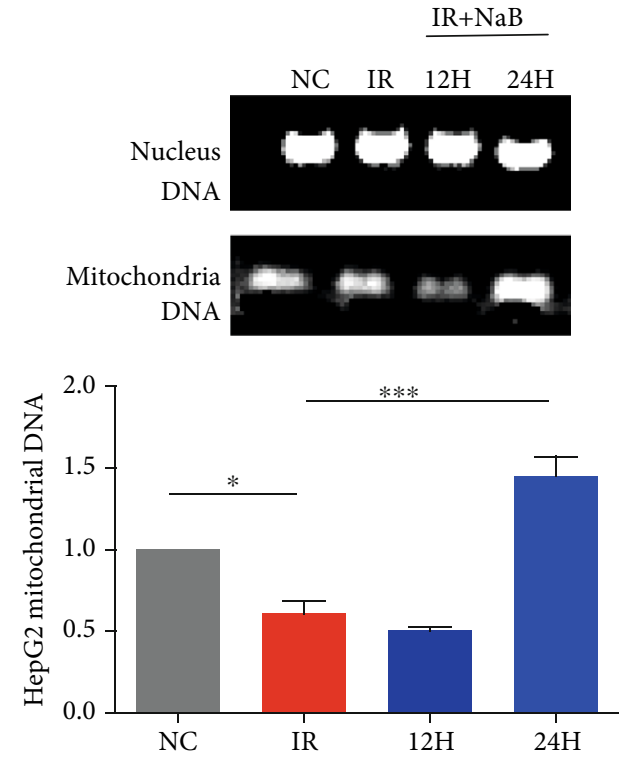

(a)

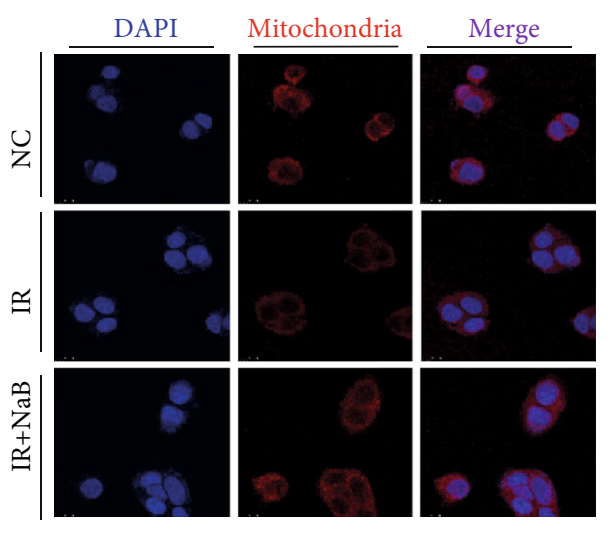

(b)

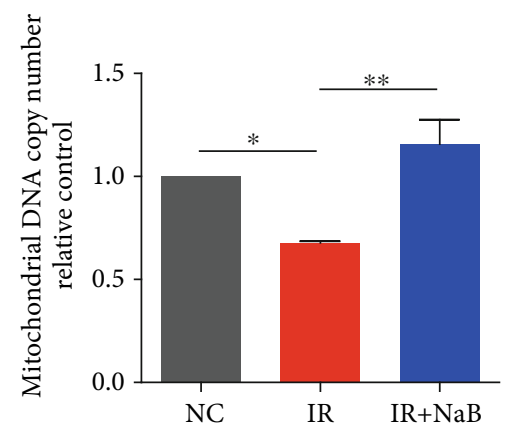

(c)
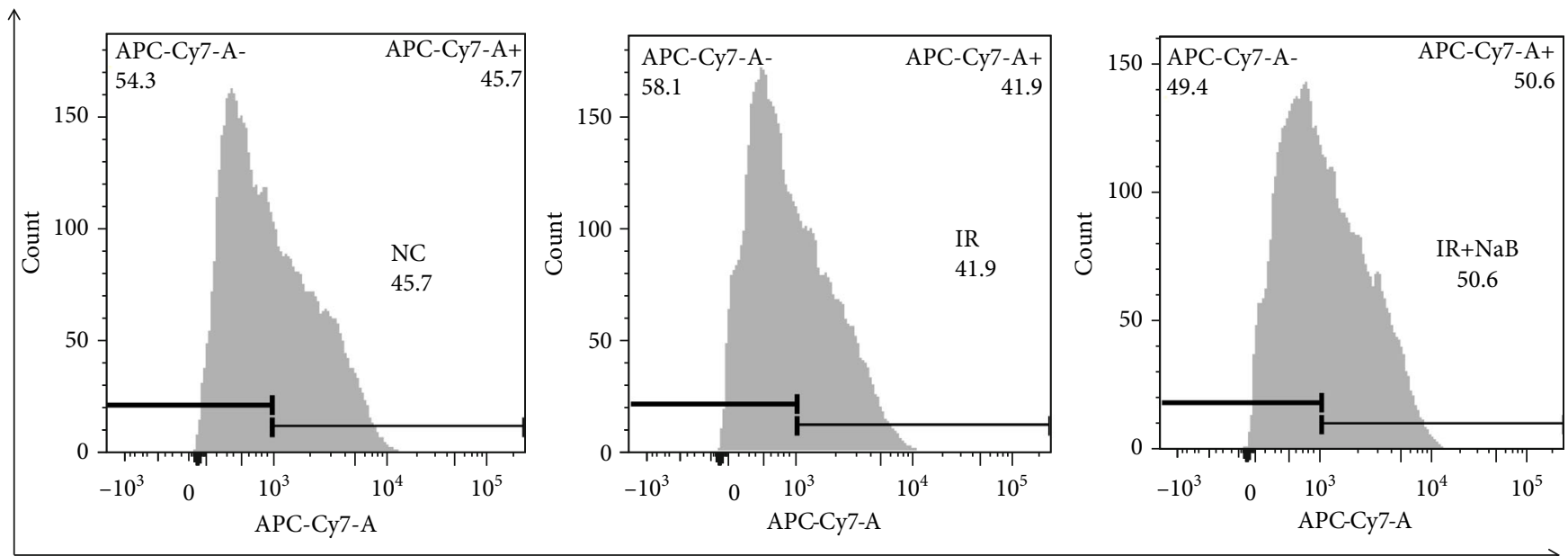

(d)

Figure 2: Continued. 


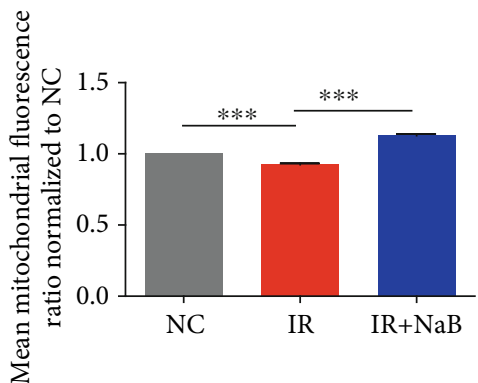

(e)
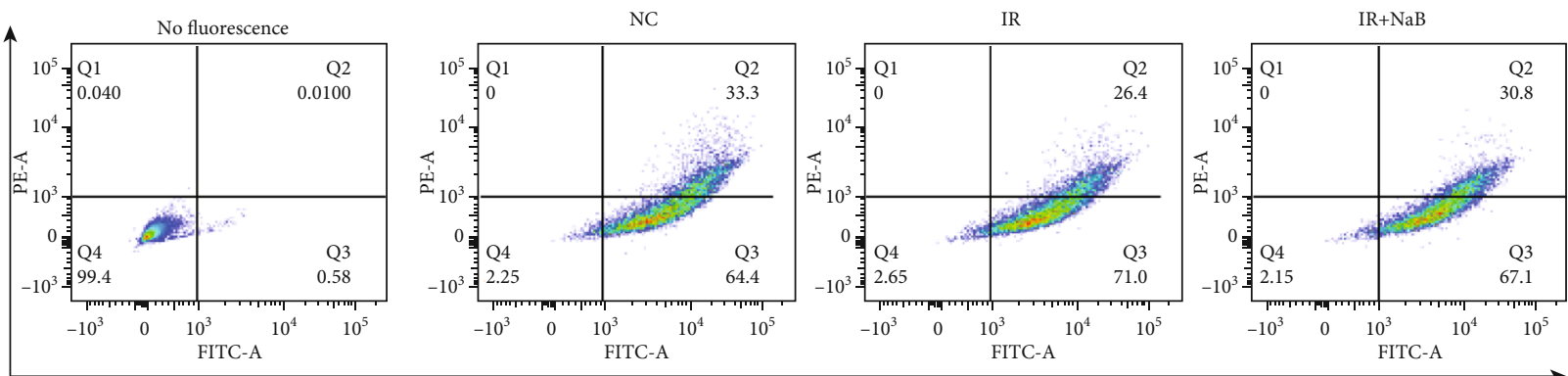

(f)

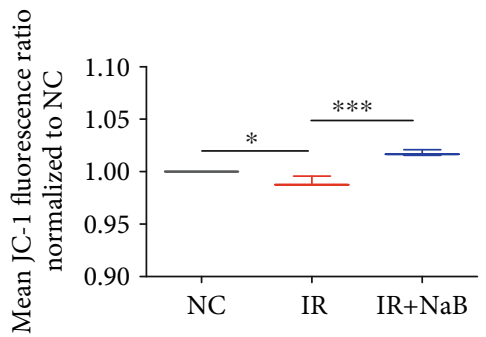

(g)

FIGURE 2: Influence of $\mathrm{NaB}$ treatment on function of mitochondria. (a) NaB increased content of mitochondrial DNA. (b) Mitochondria were observed by immunofluorescence method (magnification: 1200). Number of copies for mitochondrial DNA was analyzed by (c) Q-PCR and (d, e) flow cytometry. (f, g) Mitochondrial membrane potentials were determined by flow cytometry. NC: normal control; IR: high insulininduced insulin resistance; NaB: sodium butyrate. ${ }^{*} p<0.05,{ }^{* *} p<0.01$, and ${ }^{* * *} p<0.001$.

analyzed by the one-way ANOVA method. Comparisons between two groups were made using Student's $t$-test. $p<$ 0.05 was considered as statistically significant.

\section{Results}

3.1. Sodium Butyrate (NaB) Modulated Genes Related with Mitochondrial Energy Metabolism. Previously, we have demonstrated that $\mathrm{NaB}$ promoted glycogen metabolism within hepatocytes [8] and decreased the glucose level in $\mathrm{db} / \mathrm{db}$ mice [7]. As mitochondria play a pivotal role in modulating energy balance, we further carried out experiments to investigate influence of $\mathrm{NaB}$ on mitochondria under insulin resistance (IR) circumstances. To this end, we firstly determined changes of gene expression related with mitochondrial energy metabolism by a PCR array kit. As shown in Table 2 and Figures 1(a)-1(c), 35 genes were downregulated and 19 genes were upregulated in NaB-incubated cells compared with the model group (high insulin); among these genes, UQCRC1 was upregulated by as high as 90-folds, while COX6C was downregulated by 0.63 -fold.
TABLE 3: ATP production among groups $(n=3)$.

\begin{tabular}{lc}
\hline Group & ATP (nM/mg prot) \\
\hline NC & $349.39 \pm 38.32$ \\
IR & $250.24 \pm 3.13^{* *}$ \\
IR $+\mathrm{NaB}$ & $333.15 \pm 51.77^{\#}$ \\
\hline
\end{tabular}

NC: normal control; IR: high insulin-induced insulin resistance; $\mathrm{NaB}$ : sodium butyrate. ${ }^{* *} p<0.01$ vs. NC; ${ }^{*} p<0.05$ vs. IR.

To predict possible mechanism and signaling pathways, protein-protein interaction among genes was generated from the STRING database. As depicted in Figure 1(d), AKT and AMPK signaling pathways play a pivotal role in modulating the top ten changed genes within the mitochondria, and receptors for short chain fatty acids (SCFAs) may influence the balance of AKT and AMPK pathways.

3.2. Mitochondrial Function Was Enhanced by NaB. To investigate role of $\mathrm{NaB}$ on mitochondria, we firstly 


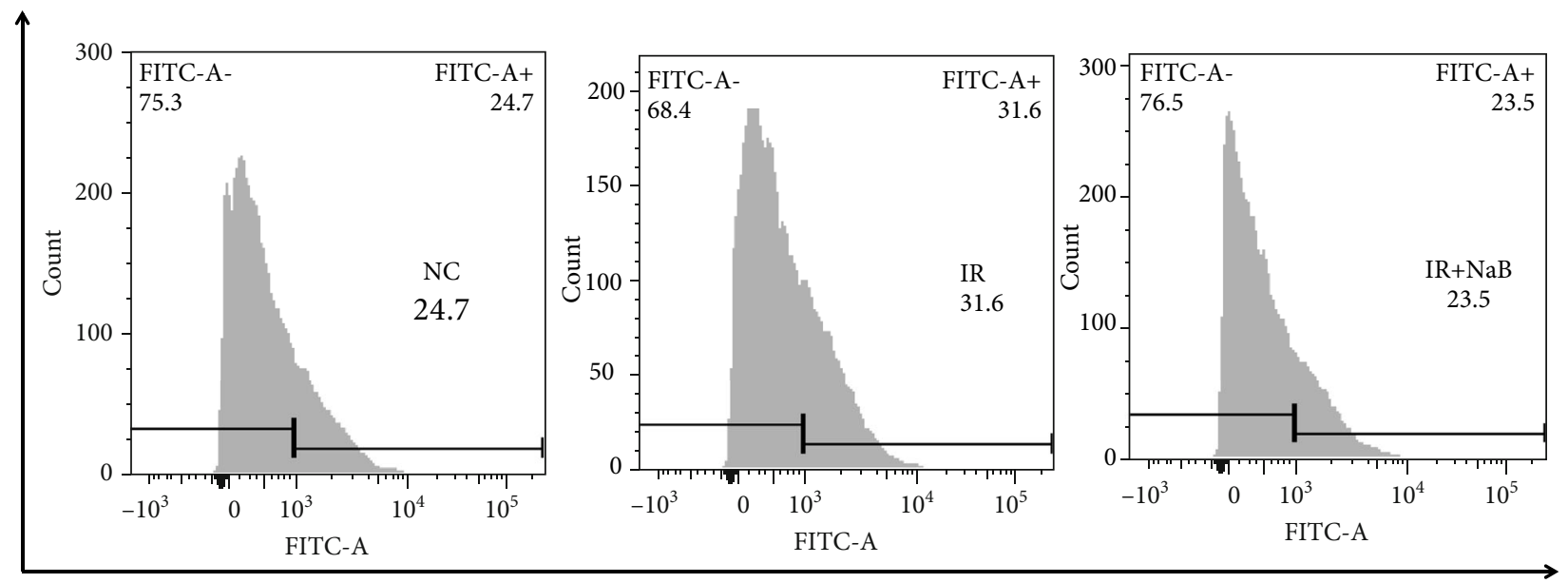

(a)

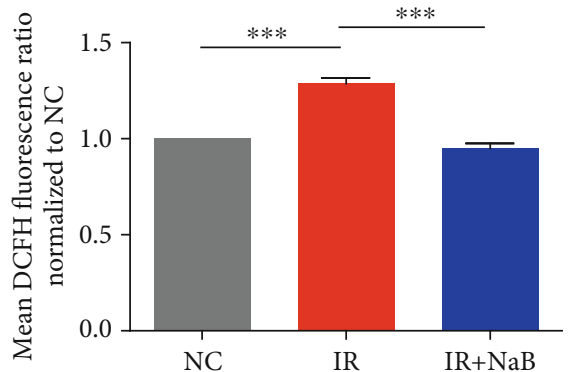

(b)
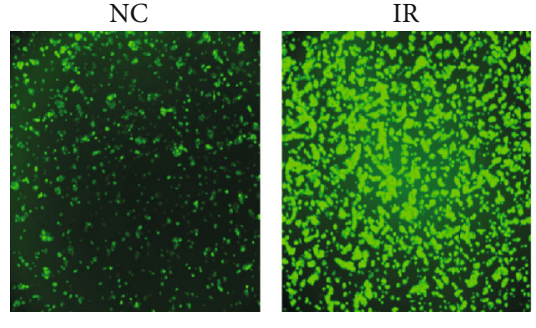

(c)

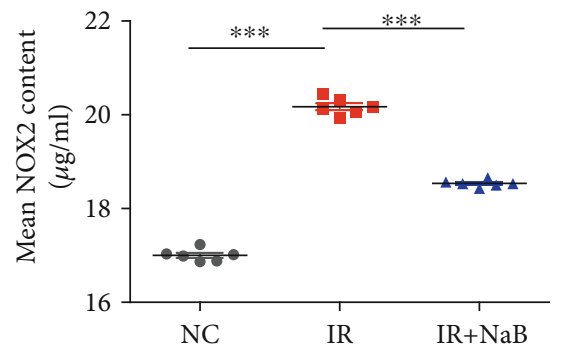

(d)

Figure 3: $\mathrm{NaB}$ ameliorated oxidative stress under high insulin settings. ROS was determined by $(\mathrm{a}, \mathrm{b})$ flow cytometer and (c) observed under a fluorescence microscope (magnification: 20). (d) Level of NOX2 was analyzed by EIA. NC: normal control; IR: high insulin-induced insulin resistance; NaB: sodium butyrate. ${ }^{* * *} p<0.001$.

determined its DNA. As shown in Figure 2(a), content of mitochondrial DNA (mtDNA) was significantly reduced by high insulin (IR), administration with $\mathrm{NaB}$ dramatically increased its level ( $p<0.001$ vs. IR), and the most significant effect was observed at $24 \mathrm{~h}$. By immunofluorescence assay (Figure 2(b)), PCR determination (Figure 2(c)), and flow cytometry assay (Figures 2(d) and 2(e)), we confirmed that the content and copy number of mtDNA were significantly increased by $\mathrm{NaB}$ treatment. More importantly, mitochondrial membrane potential as probed by JC-1 was significantly elevated (Figures 2(f) and 2(g)), and ATP production was enhanced (Table 3). The above findings demonstrated that administration with $\mathrm{NaB}$ could significantly reverse high insulin-induced hepatocyte dysfunction by promoting the function of mitochondria.
TABLE 4: Activity of enzymes participating in oxidative stress among groups $(n=3)$.

\begin{tabular}{lccc}
\hline Group & $\begin{array}{c}\text { SOD } \\
(\mathrm{mU} / \mathrm{mg} \text { prot })\end{array}$ & $\begin{array}{c}\text { GPX } \\
(\mathrm{mU} / \mathrm{mg} \text { prot })\end{array}$ & $\begin{array}{c}\text { MDA } \\
(\mathrm{nM} / \mathrm{mg} \text { prot })\end{array}$ \\
\hline $\mathrm{NC}$ & $222.80 \pm 1.23$ & $59.32 \pm 4.11$ & $1.93 \pm 0.14$ \\
$\mathrm{IR}$ & $184.12 \pm 8.99^{* *}$ & $51.88 \pm 2.25^{* *}$ & $3.35 \pm 0.1^{* *}$ \\
$\mathrm{IR}$ & $208.10 \pm 16.87^{\# \#}$ & $68.86 \pm 9.02^{\# \#}$ & $2.24 \pm 0.04^{\# \#}$ \\
$+\mathrm{NaB}$ & & & \\
\hline
\end{tabular}

NC: normal control; IR: high insulin-induced insulin resistance; $\mathrm{NaB}$ : sodium butyrate. ${ }^{* *} p<0.01$ vs. NC; ${ }^{\# *} p<0.01$ vs. IR.

3.3. NaB Ameliorated Oxidative Stress Damage under High Insulin Circumstances. Mitochondria are the major source of reactive oxygen species (ROS), and accumulation of ROS 


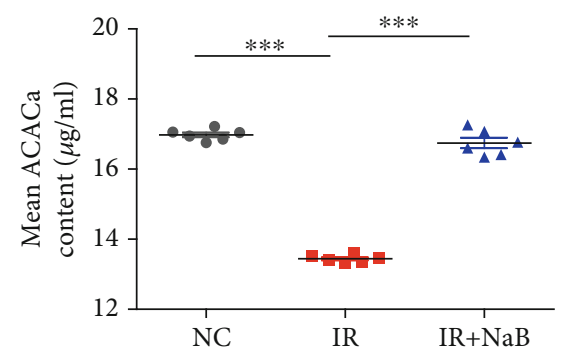

(a)

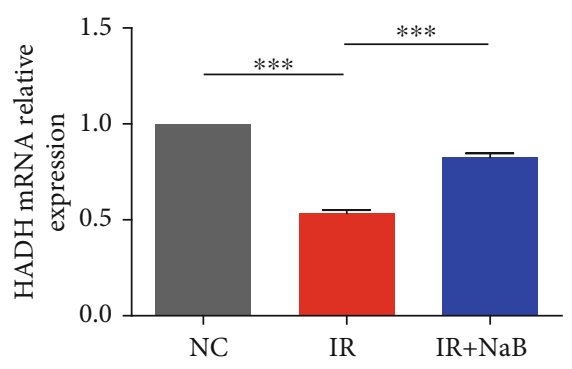

(c)

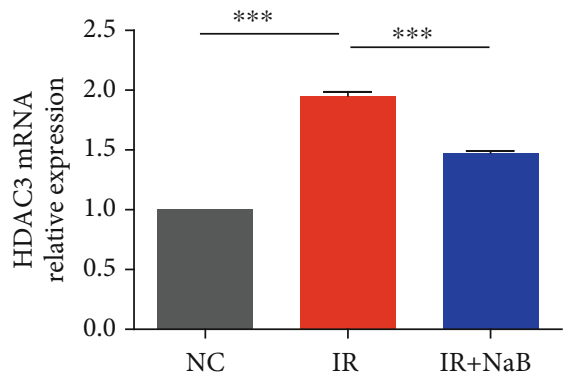

(e)

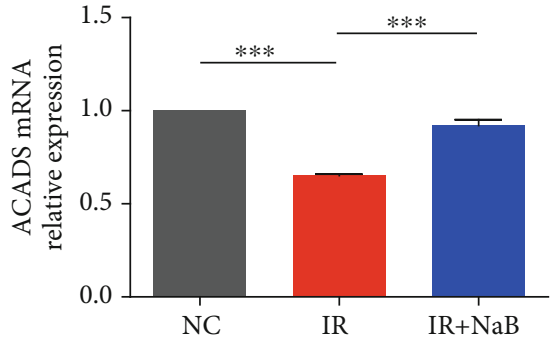

(b)

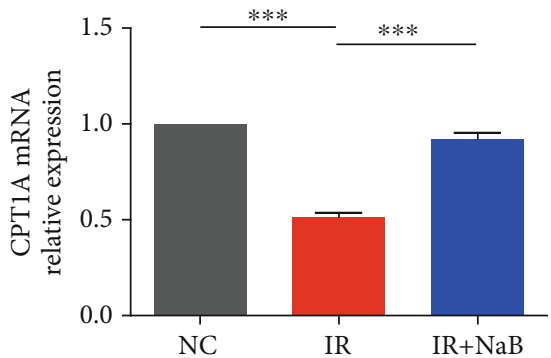

(d)

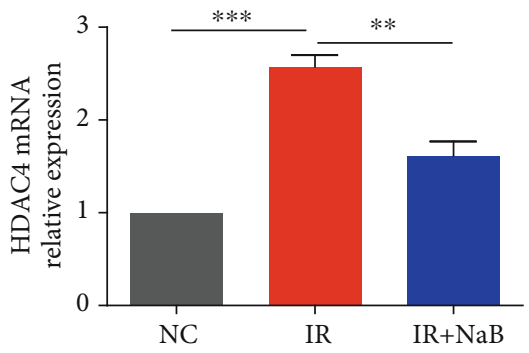

(f)

FIGURE 4: NaB modulated $\beta$-oxidation of fatty acids and histone acetylation. (a) Level of ACACa was determined by EIA. Expressions of (b) ACADS, (c) HADH, (d) CPT1A, (e) HDAC3, and (f) HDAC4 were analyzed by Q-PCR. NC: normal control; IR: high insulin-induced insulin resistance; $\mathrm{NaB}$ : sodium butyrate. ${ }^{* *} p<0.01$ and ${ }^{* * *} p<0.001$.

will lead to decreased mitochondrial membrane potential and ATP production [9]. To evaluate oxidative stress after $\mathrm{NaB}$ administration, we determined the level of ROS by a flow cytometer (Figures 3(a) and 3(b)) and observed its content under a fluorescence microscope (Figure 3(c)); we found that insulin resistance (IR) is accompanied by overproduction of ROS, and $\mathrm{NaB}$ can significantly inhibit this elevation. NADPH oxidase 2 (NOX2) within mitochondria plays a pivotal role in the production of ROS. In the present study, $\mathrm{NaB}$ dramatically inhibited activity of NOX2 induced by IR (Figure 3(d)); other enzymes and products within hepatocytes including antioxidative SOD and GPX and prooxidative MDA were also ameliorated by $\mathrm{NaB}$ (Table 4).

3.4. NaB Mediated $\beta$-Oxidation of Fatty Acids and Histone Acetylation in Hepatocytes. Acetyl-CoA carboxylase alpha (ACACa) is the rate-limiting enzyme in fatty acid synthesis and is believed to be a novel target for endocrine disease, e.g., diabetes and obesity. In our present study, the level of ACACa was dramatically reduced by high insulin and $\mathrm{NaB}$ incubation significantly increased its content to the normal level (Figure 4(a)). CPT1A, HADH, and ACADS are pivotal rate-limiting enzymes in fatty acid catabolism within mitochondria during the $\beta$-oxidation process [10]. We found that high insulin significantly inhibited their mRNA expression, while this was reversed by $\mathrm{NaB}$ administration (Figures 4(b)-4(d)). In this sense, NaB application modulated the metabolism of fatty acids within hepatocytes and exhibited protective effects on the function of the mitochondrial electron transfer chain under high insulin circumstances.

Histone deacetylase (HDAC) modulates deacetylation modification of histones, thus inhibiting gene translocation and thereafter energy metabolism. Activities of rate-limiting enzymes discussed above are modulated by both the histone acetylation level and deacetylase activity. HDAC3 and HDAC4 are typical HDACs that belong to class I and II HDACs, respectively, and loss of HDAC in the liver will result in increased glycogen storage and reduced blood glucose level [11]. In the present study, we found that $\mathrm{NaB}$ significantly inhibited the expression of HDAC3 and 4 induced by high insulin (Figures 4(e) and 4(f)). 


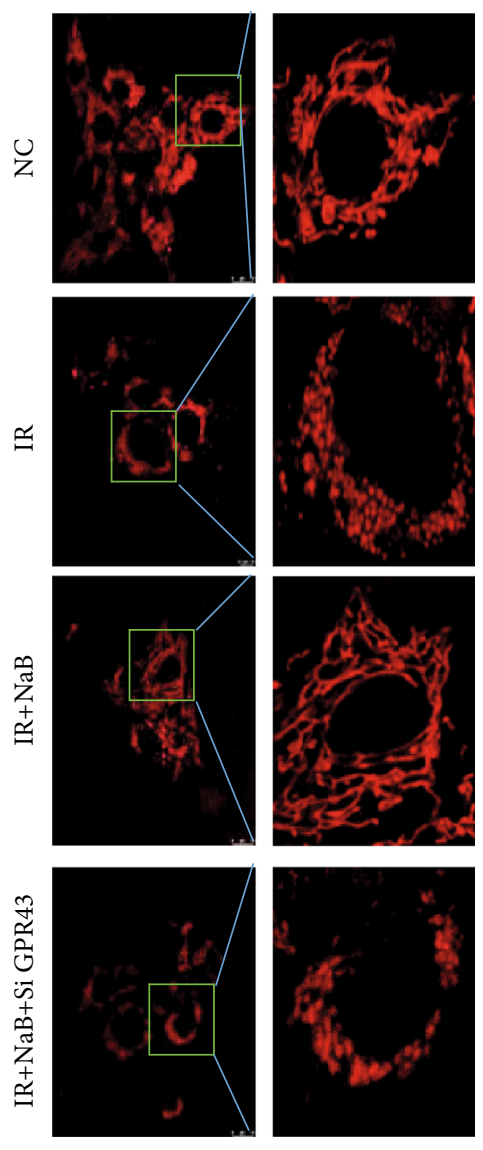

(a)

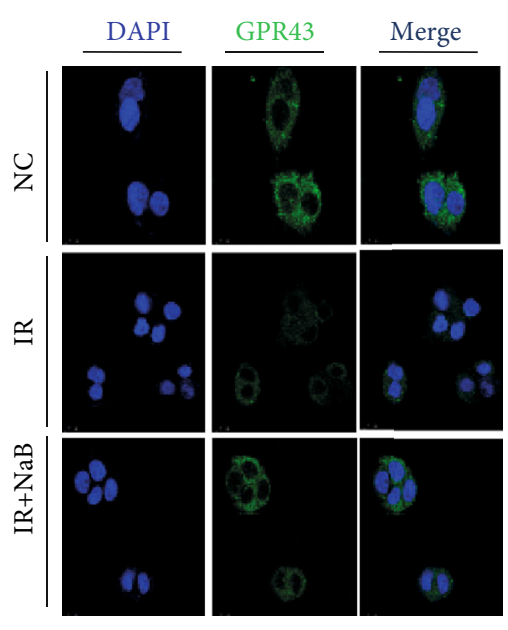

(b)

DAPI $\quad \beta$-Arrestin2 Merge

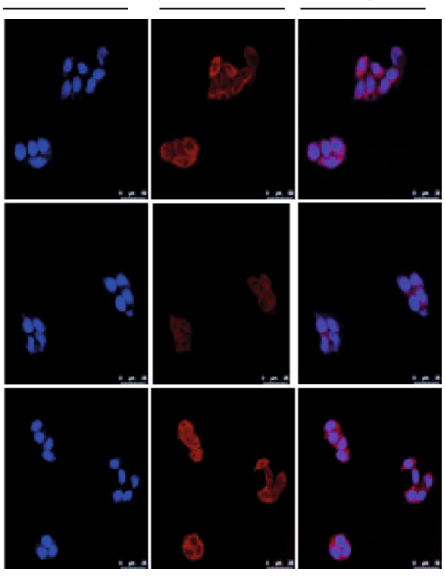

(d)

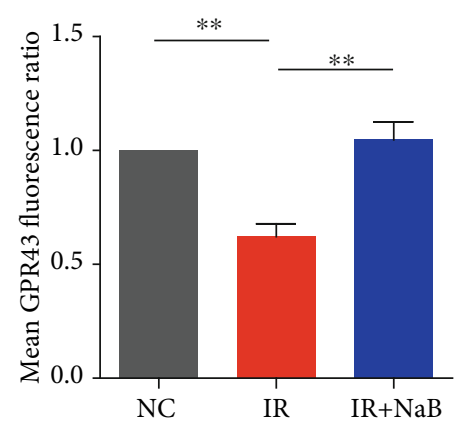

(c)

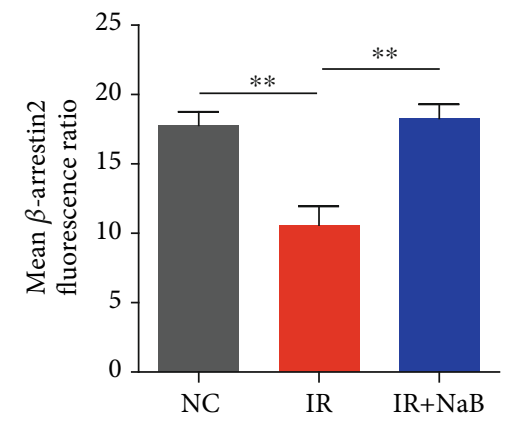

(e)

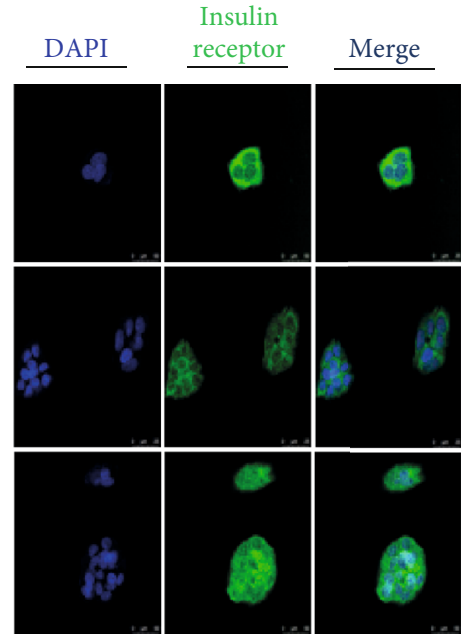

(f)

Figure 5: Continued. 


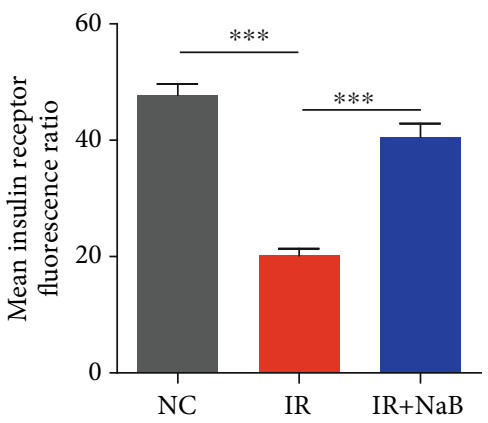

(g)

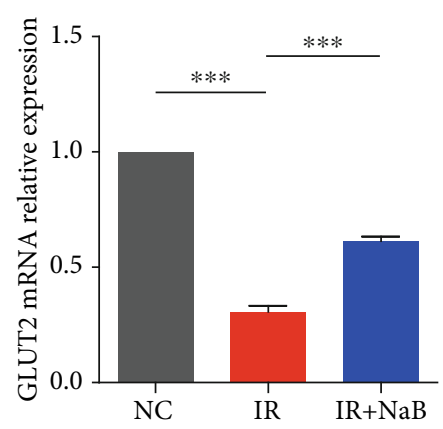

(h)

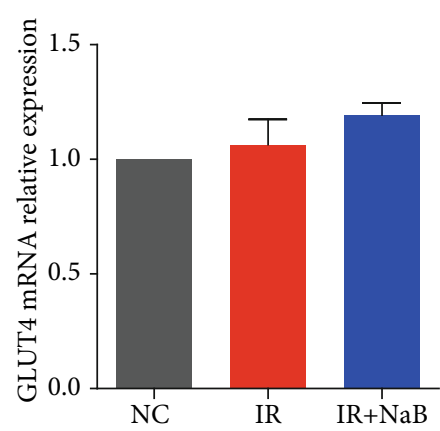

(i)

FIgURE 5: $\mathrm{NaB}$ increased expression of signal transduction proteins on cell membranes. (a) Shape of mitochondria within live cells were stained with MitoTracker ${ }^{\mathrm{TM}}$ Deep Red FM and observed under a confocal microscope (magnification: 1200). Expression and location for $(b, c)$ GPR43, (d, e) beta-arrestin2, and (f, g) insulin receptor were determined by immunofluorescence and observed under a confocal microscope (magnification: 800). mRNA expression of (h) GLUT2 and (i) GLUT4 was determined by Q-PCR. NC: normal control; IR: high insulin-induced insulin resistance; NaB: sodium butyrate. ${ }^{* *} p<0.01$ and ${ }^{* * *} p<0.001$.

\subsection{GPR43 Mediated Function of NaB on Mitochondria.} Previously, we have demonstrated that GPR43 mediated the function of $\mathrm{NaB}$ on glycogen metabolism within the hepatocyte [8]. To explore the underlying mechanism of $\mathrm{NaB}$ on mitochondria, we firstly knocked down the expression of GPR43 by siRNA and observed its influence on the shape and distribution of mitochondria under a confocal microscope. As shown in Figure 5(a), high insulin (IR) induced an obvious fragmentation of mitochondria; $\mathrm{NaB}$ incubation significantly reversed the shape change of mitochondria via GPR43. This was further demonstrated by an immunofluorescence assay that $\mathrm{NaB}$ significantly increased the expression of GPR43 that was inhibited by IR (Figures 5(b) and 5(c)).

There is a previous report which indicated that $\beta$ arrestin2 mediated internalization of GPR43 [12], and its expression in diabetic mice was dramatically reduced. In the present study, we observed that $\mathrm{NaB}$ application significantly induced the expression of $\beta$-arrestin2 within hepatocytes (Figures 5(d) and 5(e)); more importantly, expression of the insulin receptor was also upregulated by $\mathrm{NaB}$ (Figures 5(f) and 5(g)). This was consistent with proteinprotein interaction prediction from the STRING database (Figure 1(d)).

In the current study, we also observed mRNA upregulation of GLUT2 (Figure 5(h)) but not GLUT4 (Figure 5(i)) by $\mathrm{NaB}$ incubation under high insulin circumstances. This is in line with our previous findings [8], suggesting the $\mathrm{NaB}$-promoted entrance of glucose into the cells may benefit energy metabolism within mitochondria.

3.6. AMPK-PGC1-alpha Signaling Pathways Modulated Effects of $\mathrm{NaB}$ on Mitochondria. The AKT signaling pathway plays a pivotal role in modulating glucose uptake and metabolism within mitochondria. We found that high insulin significantly induced activation of AKT while reducing p-GSK3 compared with the normal control $(p<0.001)$, and $\mathrm{NaB}$ reversed this trend to the normal levels (Figures 6(a)6(d)). On the other hand, the AMPK-PGC1-alpha signaling pathway, which modulates both biogenesis and function of mitochondria, was significantly enhanced on application of $\mathrm{NaB}$ (Figures 6(e)-6(i)).

\section{Discussion and Conclusions}

Insulin resistance in hepatocytes is one of the central reasons that block glucose metabolism. Recent findings have indicated the important role of cometabolism between gut microbiota and the organism. But the underlying mechanism is still not fully understood. In the current study, we demonstrated that a metabolite product from gut microbiota, sodium butyrate $(\mathrm{NaB})$, can ameliorate function of hepatocytes via modulating mitochondrial metabolism.

According to a report from Kanazawa and colleagues [1], as high as $19 \%$ cases with type 2 diabetes (T2D) are accompanied with liver dysfunction. Concerning the pivotal role of the liver in mediating the metabolism of glucose and lipids, preserving its function helps to inhibit progression of T2D. With the understanding of the influence of gut microbiota towards preserving the organism in a healthy status, effects of the metabolites from microbiota against disease development have attracted more attention. It was found by Qin and colleagues that butyrate-producing bacteria were significantly reduced in a T2D population [4]. Although physiological concentration of butyrate within the liver is low, external administration with butyrate has been suggested to fight against high-fat diet-induced fatty liver [5]; this also suggested potential effects of butyrate against the development of T2D. Previously, we demonstrated in $\mathrm{db} / \mathrm{db}$ mice that oral administration with $\mathrm{NaB}$ could significantly reduce HbA1c and diabetic inflammation [7]; more importantly, hypertrophy and steatosis of hepatocytes in $\mathrm{db} / \mathrm{db}$ mice were significantly reversed by $\mathrm{NaB}$, accompanied with enhancement of glycogen metabolism [8]. Our findings are in line with a report from Khan and Jena that $\mathrm{NaB}$ inhibited liver vascular steatosis and fat deposition [6]. But the underlying mechanism still needs to be fully explored.

Diabetes is closely related with significantly reduced mitochondrial function. In the diabetic population, mitochondrial numbers are found to be reduced [2], lipid oxidation is significantly impaired [3], and a direct relationship between mitochondria and insulin resistance is exhibited $[2,13]$. To explore the relationship between $\mathrm{NaB}$ and liver function, we firstly carried out a PCR array assay to observe 


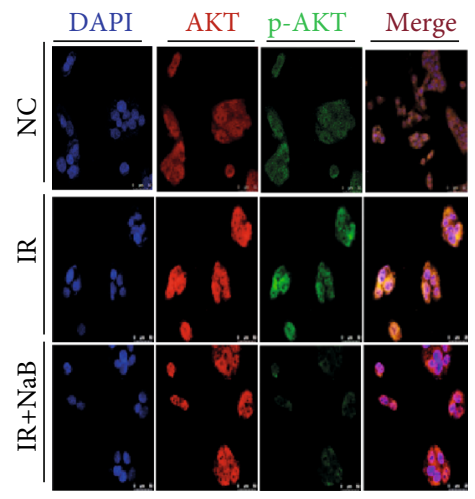

(a)

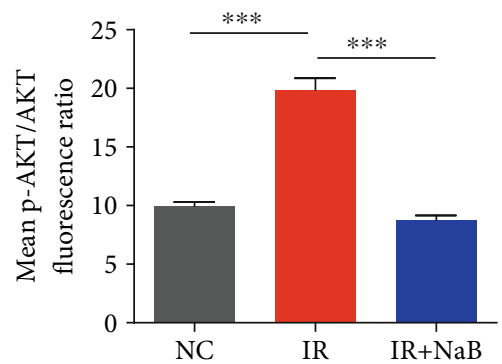

(b)

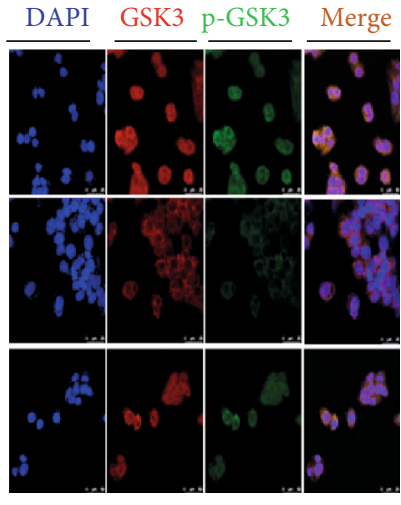

(c)

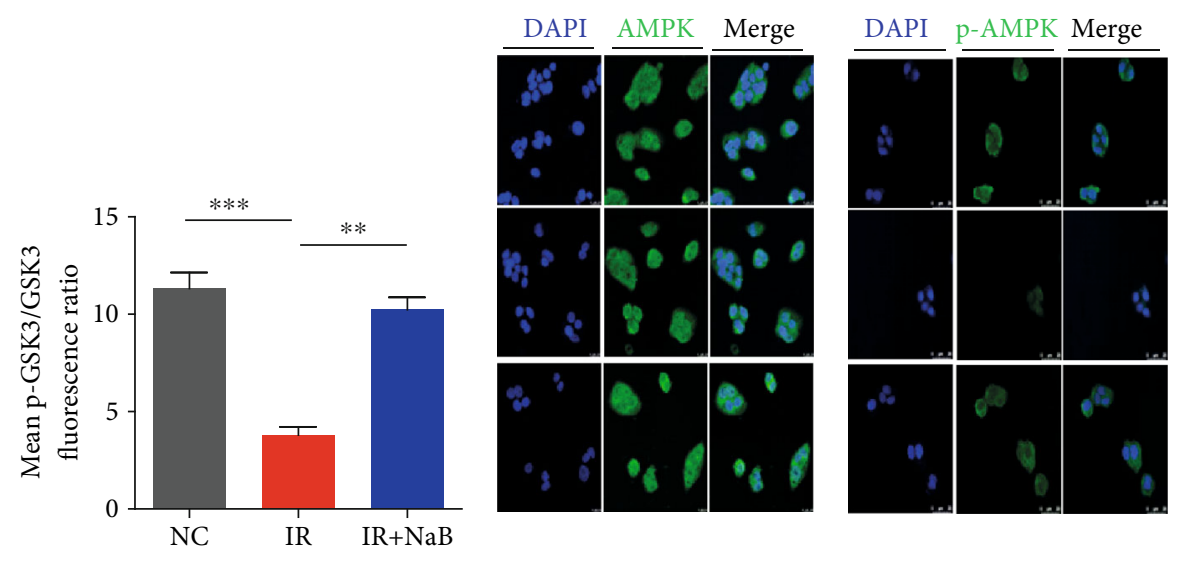

(d)

(e)

(f)

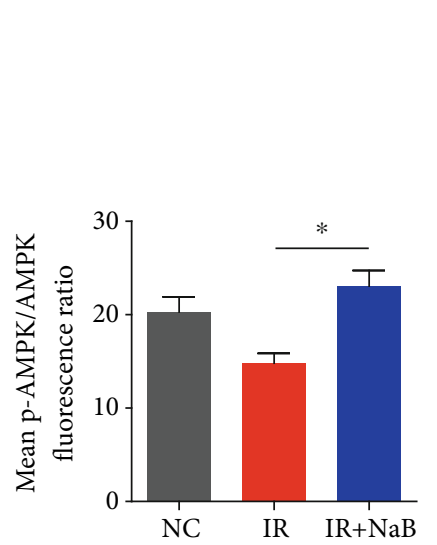

(g)

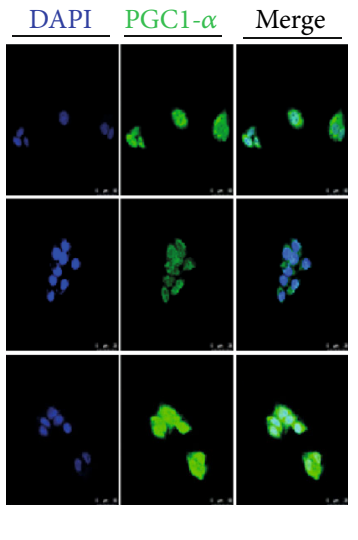

(h)

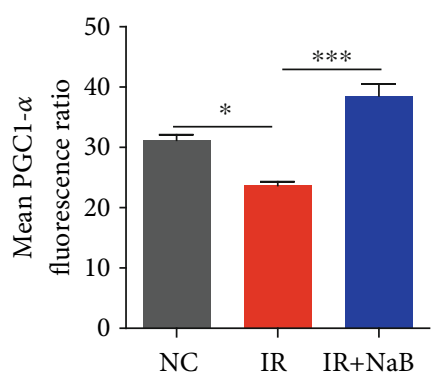

(i)

FIGURE 6: AKT and AMPK signaling pathways participated in effects of NaB on mitochondria. Expression and activation of (a, b) pAKT/AKT, (c, d) p-GSK3/GSK3, (e-g) p-AMPK/AMPK, and (h, i) PGC1- $\alpha$ were observed by immunofluorescence method (magnification: 800). NC: normal control; IR: high insulin-induced insulin resistance; NaB: sodium butyrate. ${ }^{*} p<0.05,{ }^{* *} p<0.01$, and ${ }^{* * *} p<0.001$.

changes in gene expression. We found that $\mathrm{NaB}$ administration significantly increased 19 genes while downregulating as many as 35 genes in mitochondria. As most of these genes encode and regulate the composition and function of mitochondria, we further predicted protein-protein interaction between these genes and pathways related with short chain fatty acids by the STRING database. We found that there exists a possible direct relationship between short chain fatty acids and mitochondria, and the content of mitochondria and AMPK pathways is a possible reason that contributes to this relationship.

In the present study, we incubated HepG2 cell with relatively high concentration of insulin to induce insulin resistance. We found that high insulin significantly reduced both the amount and the copy of mitochondrial DNA, and mitochondrial membrane potential was decreased, while 


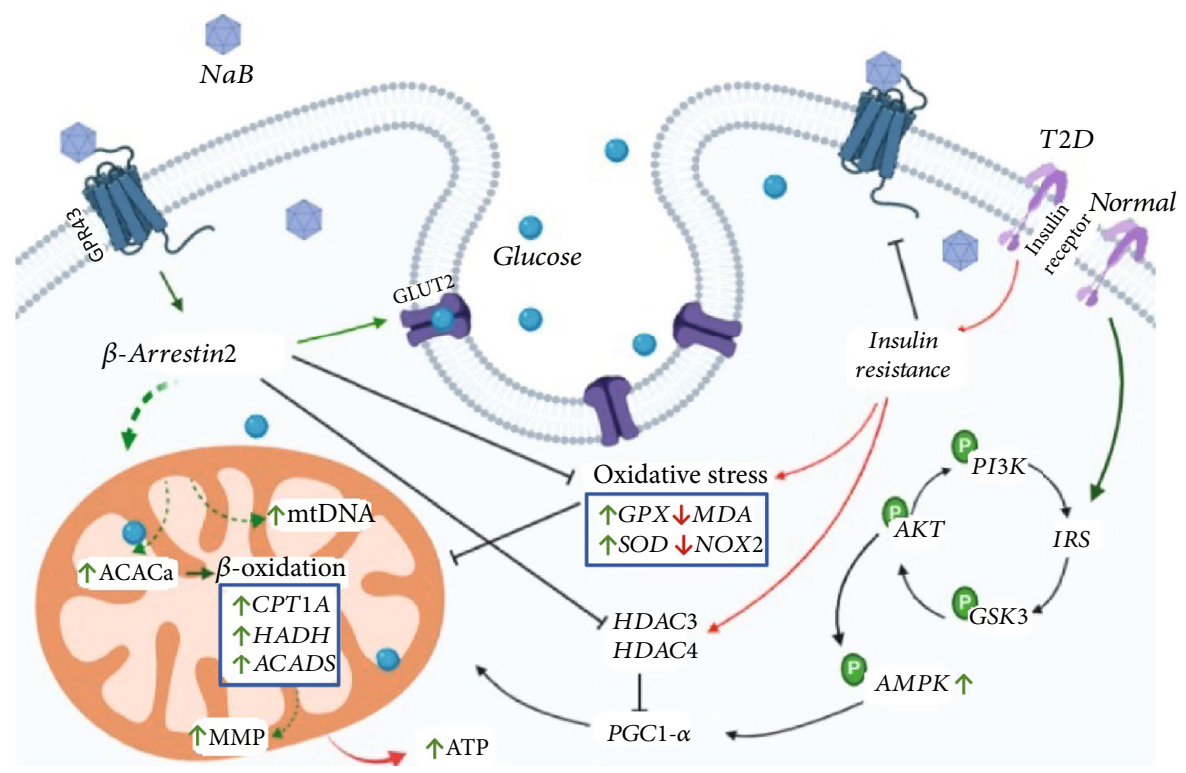

FIgURE 7: Mechanism of sodium butyrate $(\mathrm{NaB})$ on modulating high insulin-induced dysfunction of mitochondria within hepatocytes. T2D: type 2 diabetes; MMP: mitochondrial membrane potential.

application with $\mathrm{NaB}$ significantly increased mitochondrial DNA and elevated the membrane potential. Our present findings suggest that $\mathrm{NaB}$ could increase the content of mitochondria and ameliorate its dysfunction.

Inevitable by-products of mitochondrial respiration are reactive oxygen species (ROS). In fact, mitochondria themselves contribute to the main production of ROS. Amounts of studies have demonstrated that overaccumulation of ROS and oxidative stress is one of the characteristic of diabetes. Excessive ROS in the absence of sufficient antioxidants will lead to extensive production of oxidative by-products and events, such as generation and accumulation of advanced glycation end products (AGEs), the damage of both nuclear and mitochondrial DNA (mtDNA) [14], and even cell death. Suppression of oxidative stress has been demonstrated to benefit diabetes management. SOD and GPX are two representative antioxidation enzymes. Overexpression of SOD significantly ameliorated insulin resistance in highfat diet mice [15]. It was interesting in our present study that $\mathrm{NaB}$ increased both SOD and GPX expressions and decreased prooxidative NOX2, ROS, and MDA levels. This finding obviously demonstrated that $\mathrm{NaB}$ enhanced the function of mitochondria but did not increase the risk of oxidative stress damage.

In fact, production of ATP within mitochondria relies on oxidation. CPT1A, HADH, and ACADS are pivotal rate-limiting enzymes in fatty acid catabolism within mitochondria during $\beta$-oxidation [10], and their activities are modulated by histone acetylation and deacetylation. Histone deacetylase (HDAC) directly controls deacetylation modification of histones, and loss of HDAC in the liver will result in increased glycogen storage and reduced blood glucose level [11]. It has been demonstrated that HDAC protein coprecipitated with CPT1A [16]. HDAC3 and HDAC4 are typical HDACs that belong to class I and II HDACs, respectively. Reports indicated that class I HDAC contributed to mitochondrial dysfunction [17] and treatment with its inhibitor promoted energy expenditure and reduced both glucose and insulin levels by increasing PGC-1alpha activity [18]. In the present study, we observed that high insulin significantly inhibited expressions of ratelimiting enzymes of oxidation including ACACs, CPT1A, $\mathrm{HADH}$, and ACADS, while their upstream HDAC was elevated, suggesting the mitochondrial electron transfer chain was blocked under high insulin settings, and $\mathrm{NaB}$ application ameliorated their expression. There is a previous study that demonstrated that short chain fatty acids (SCFAs), including $\mathrm{NaB}$, possess a natural inhibitory effect on HDAC [19]. In this sense, $\mathrm{NaB}$ may modulate oxidation within mitochondria via inhibiting HDAC.

The GPR43- $\beta$-arrestin2 pathway has been demonstrated to mediate the function of $\mathrm{NaB}[8,12]$. GPR43 is a $\mathrm{G}$ protein-coupled protein on the cell membrane, and $\beta$ arrestin 2 is one of its downstream activators that are usually recognized as a modulator of inflammation. A report has demonstrated that deficiency of $\beta$-arrestin 2 will lead to insulin resistance [2]. A most recent study from Pydi and colleagues [21] indicated the essential role of $\beta$-arrestin 2 in maintaining energy homeostasis within adipocytes. Another study also suggested the pivotal function of $\beta$-arrestin 2 for maintaining euglycemia in hepatocytes [22]. But its involvement in mitochondrial dysfunction under high insulin settings is still not clear. In this study, we demonstrated that high insulin-induced GPR43 and $\beta$-arrestin 2 reduction was significantly reversed by $\mathrm{NaB}$; more importantly, both the insulin receptor and GLUT2 were upregulated on $\mathrm{NaB}$ administration, suggesting the amelioration of insulin resistance and energy metabolism.

Mitochondrial content is influenced by its biogenesis [23], and this process is mainly regulated by peroxisome proliferator-activated receptor coactivator-1alpha (PGC1alpha) [24]. It was found in diabetic patients that the 
expression of PGC-1alpha was reduced [25], and upregulation of PGC-1alpha can significantly increase both insulin sensitivity and lipid oxidation [26]. Studies have demonstrated that phosphorylation of AMPK will activate PGC1alpha, increase expression of mitochondria-related genes [27], and promote mitochondrial biogenesis, while HDAC1 and HDAC3 have been found to repress the transcriptional activity of PGC-1alpha [28 $]^{-}[30]$. A recent report from Yoshida and colleagues demonstrated that knockdown of GPR43 will reduce SCFA-induced activation of AMPK [31]. Our findings in this study obviously suggest that $\mathrm{NaB}$ promoted the biogenesis of mitochondria via promoting AMPK-PGC1-alpha and blocking the HDAC signaling pathway.

In conclusion, we found in our present study that sodium butyrate administration could significantly promote biogenesis and function of mitochondria under high insulin circumstances, and the GPR43- $\beta$-arrestin2-AMPKPGC1-alpha signaling pathway contributed to these effects (Figure 7). Our present findings obviously provide new insight on the pivotal role of metabolites from microbiota in maintaining euglycemia.

\section{Data Availability}

Data in this paper are available on PubMed or Scopus. Any previous paper not accessible could be requested from the corresponding author.

\section{Conflicts of Interest}

There is no conflict of interest that could be perceived as prejudicing the impartiality of the research reported.

\section{Acknowledgments}

We would like to thank Ms. Lou Chi Han from Macau University of Science and Technology (Macao, China) for the kind suggestion and technical support in this study. This work is supported by the Science and Technology Development Fund of Macau, Macau SAR, China (File Nos.: 0006/2019/A, 0093/2018/A3, and 0025/2019/AGJ) and National Natural Science Foundation of China (81873270).

\section{References}

[1] I. Kanazawa, K. Tanaka, and T. Sugimoto, "DPP-4 inhibitors improve liver dysfunction in type 2 diabetes mellitus," Medical Science Monitor, vol. 20, pp. 1662-1667, 2014.

[2] R. Boushel, E. Gnaiger, P. Schjerling, M. Skovbro, R. Kraunsøe, and F. dela, "Patients with type 2 diabetes have normal mitochondrial function in skeletal muscle," Diabetologia, vol. 50, no. 4, pp. 790-796, 2007.

[3] M. E. Patti, A. J. Butte, S. Crunkhorn et al., "Coordinated reduction of genes of oxidative metabolism in humans with insulin resistance and diabetes: potential role of PGC1 and NRF1," Proceedings of the National Academy of Sciences of the United States of America, vol. 100, no. 14, pp. 8466-8471, 2003.
[4] J. Qin, Y. Li, Z. Cai et al., "A metagenome-wide association study of gut microbiota in type 2 diabetes," Nature, vol. 490, no. 7418, pp. 55-60, 2012.

[5] G. Mattace Raso, R. Simeoli, R. Russo et al., "Effects of sodium butyrate and its synthetic amide derivative on liver inflammation and glucose tolerance in an animal model of steatosis induced by high fat diet," PLoS One, vol. 8, no. 7, article e68626, 2013.

[6] S. Khan and G. Jena, "Sodium butyrate reduces insulin-resistance, fat accumulation and dyslipidemia in type-2 diabetic rat: a comparative study with metformin," Chemico-Biological Interactions, vol. 254, pp. 124-134, 2016.

[7] Y. H. Xu, C. L. Gao, H. L. Guo et al., "Sodium butyrate supplementation ameliorates diabetic inflammation in $\mathrm{db} / \mathrm{db}$ mice," The Journal of Endocrinology, vol. 238, no. 3, pp. 231-244, 2018.

[8] W. Q. Zhang, T. T. Zhao, D. K. Gui et al., "Sodium butyrate improves liver glycogen metabolism in type 2 diabetes mellitus," Journal of Agricultural and Food Chemistry, vol. 67, no. 27, pp. 7694-7705, 2019.

[9] D. B. Zorov, M. Juhaszova, and S. J. Sollott, "Mitochondrial reactive oxygen species (ROS) and ROS-induced ROS release," Physiological Reviews, vol. 94, no. 3, pp. 909-950, 2014.

[10] S. Pucci, M. J. Zonetti, T. Fisco et al., "Carnitine palmitoyl transferase-1A (CPT1A): a new tumor specific target in human breast cancer," Oncotarget, vol. 7, no. 15, pp. 1998219996, 2016.

[11] M. M. Mihaylova, D. S. Vasquez, K. Ravnskjaer et al., "Class IIa histone deacetylases are hormone-activated regulators of FOXO and mammalian glucose homeostasis," Cell, vol. 145, no. 4, pp. 607-621, 2011.

[12] S. U. Lee, H. J. in, M. S. Kwon et al., " $\beta$-Arrestin 2 mediates G protein-coupled receptor 43 signals to nuclear factor- $\kappa \mathrm{B}$," Biological \& Pharmaceutical Bulletin, vol. 36, no. 11, pp. 1754-1759, 2013.

[13] D. E. Kelley, K. V. Williams, and J. C. Price, "Insulin regulation of glucose transport and phosphorylation in skeletal muscle assessed by PET," The American Journal of Physiology, vol. 277, no. 2, pp. E361-E369, 1999.

[14] F. Song, W. Jia, Y. Yao et al., "Oxidative stress, antioxidant status and DNA damage in patients with impaired glucose regulation and newly diagnosed type 2 diabetes," Clinical Science, vol. 112, no. 12, pp. 599-606, 2007.

[15] K. L. Hoehn, A. B. Salmon, C. Hohnen-Behrens et al., "Insulin resistance is a cellular antioxidant defense mechanism," Proceedings of the National Academy of Sciences of the United States of America, vol. 106, no. 42, pp. 1778717792, 2009.

[16] P. Mazzarelli, S. Pucci, E. Bonanno, F. Sesti, M. Calvani, and L. G. Spagnoli, "Carnitine palmitoyltransferase I in human carcinomas: a novel role in histone deacetylation?," Cancer Biology \& Therapy, vol. 6, no. 10, pp. 1606-1613, 2007.

[17] B. Lkhagva, Y. H. Kao, T. I. Lee, T. W. Lee, W. L. Cheng, and Y. J. Chen, "Activation of class I histone deacetylases contributes to mitochondrial dysfunction in cardiomyocytes with altered complex activities," Epigenetics, vol. 13, no. 4, pp. 376-385, 2018.

[18] A. Galmozzi, N. Mitro, A. Ferrari et al., "Inhibition of class I histone deacetylases unveils a mitochondrial signature and enhances oxidative metabolism in skeletal muscle and adipose tissue," Diabetes, vol. 62, no. 3, pp. 732-742, 2013. 
[19] M. Göttlicher, S. Minucci, P. Zhu et al., "Valproic acid defines a novel class of HDAC inhibitors inducing differentiation of transformed cells," The EMBO Journal, vol. 20, no. 24, pp. 6969-6978, 2001.

[20] B. Luan, J. Zhao, H. Wu et al., "Deficiency of a beta-arrestin-2 signal complex contributes to insulin resistance," Nature, vol. 457, no. 7233, pp. 1146-1149, 2009.

[21] S. P. Pydi, S. Jain, W. Tung et al., “Adipocyte $\beta$-arrestin-2 is essential for maintaining whole body glucose and energy homeostasis," Nature Communications, vol. 10, no. 1 , p. 2936, 2019.

[22] L. Zhu, M. Rossi, Y. Cui et al., "Hepatic $\beta$-arrestin 2 is essential for maintaining euglycemia," The Journal of Clinical Investigation, vol. 127, no. 8, pp. 2941-2945, 2017.

[23] D. A. Hood, L. D. Tryon, H. N. Carter, Y. Kim, and C. C. W. Chen, "Unravelling the mechanisms regulating muscle mitochondrial biogenesis," The Biochemical Journal, vol. 473, no. 15, pp. 2295-2314, 2016.

[24] C. Ploumi, I. Daskalaki, and N. Tavernarakis, "Mitochondrial biogenesis and clearance: a balancing act," The FEBS Journal, vol. 284, no. 2, pp. 183-195, 2017.

[25] V. K. Mootha, C. M. Lindgren, K. F. Eriksson et al., "PGC1alpha-responsive genes involved in oxidative phosphorylation are coordinately downregulated in human diabetes," Nature Genetics, vol. 34, no. 3, pp. 267-273, 2003.

[26] C. R. Benton, G. P. Holloway, X. X. Han et al., "Increased levels of peroxisome proliferator-activated receptor gamma, coactivator 1 alpha (PGC-1alpha) improve lipid utilisation, insulin signalling and glucose transport in skeletal muscle of lean and insulin-resistant obese Zucker rats," Diabetologia, vol. 53, no. 9, pp. 2008-2019, 2010.

[27] S. Jager, C. Handschin, J. St-Pierre, and B. M. Spiegelman, "AMP-activated protein kinase (AMPK) action in skeletal muscle via direct phosphorylation of PGC-1alpha," Proceedings of the National Academy of Sciences of the United States of America, vol. 104, no. 29, pp. 12017-12022, 2007.

[28] G. Canettieri, I. Morantte, E. Guzmán et al., “Attenuation of a phosphorylation-dependent activator by an HDAC-PP1 complex," Nature Structural Biology, vol. 10, no. 3, pp. 175-181, 2003.

[29] S. Grégoire, L. Xiao, J. Nie et al., "Histone deacetylase 3 interacts with and deacetylates myocyte enhancer factor 2," Molecular and Cellular Biology, vol. 27, no. 4, pp. 1280-1295, 2007.

[30] C. Handschin, J. Rhee, J. Lin, P. T. Tarr, and B. M. Spiegelman, "An autoregulatory loop controls peroxisome proliferatoractivated receptor gamma coactivator 1alpha expression in muscle," Proceedings of the National Academy of Sciences of the United States of America, vol. 100, no. 12, pp. 7111-7116, 2003.

[31] H. Yoshida, M. Ishii, and M. Akagawa, "Propionate suppresses hepatic gluconeogenesis via GPR43/AMPK signaling pathway," Archives of Biochemistry and Biophysics, vol. 672, article 108057, 2019. 